\title{
Bose-Einstein condensation in a frustrated triangular optical lattice
}

\author{
Peter Janzen ${ }^{1}$, Wen-Min Huang ${ }^{2, *}$, L. Mathey ${ }^{1,3, \dagger}$ \\ ${ }^{1}$ Zentrum für Optische Quantentechnologien and Institut für Laserphysik, Universität Hamburg, 22761 Hamburg, Germany \\ ${ }^{2}$ Department of Physics, National Chung-Hsing University, Taichung 40227, Taiwan \\ ${ }^{3}$ The Hamburg Centre for Ultrafast Imaging, Luruper Chaussee 149, Hamburg 22761, Germany
}

(Dated: June 21, 2021)

\begin{abstract}
The recent experimental condensation of ultracold atoms in a triangular optical lattice with a negative effective tunneling parameter paves the way to study frustrated systems in a controlled environment. Here, we explore the critical behavior of the chiral phase transition in such a frustrated lattice in three dimensions. We represent the low-energy action of the lattice system as a twocomponent Bose gas corresponding to the two minima of the dispersion. The contact repulsion between the bosons separates into intra- and inter-component interactions, referred to as $V_{0}$ and $V_{12}$, respectively. We first employ a Huang-Yang-Luttinger approximation of the free energy. For $V_{12} / V_{0}=2$, which corresponds to the bare interaction, this approach suggests a first order phase transition, at which both the U(1) symmetry of condensation and the $\mathbb{Z}_{2}$ symmetry of the emergent chiral order are broken simultaneously. Furthermore, we perform a renormalization group calculation at one-loop order. We demonstrate that the coupling regime $0<V_{12} / V_{0} \leq 1$ shares the critical behavior of the Heisenberg fixed point at $V_{12} / V_{0}=1$. For $V_{12} / V_{0}>1$ we show that $V_{0}$ flows to a negative value, while $V_{12}$ increases and remains positive. This results in a breakdown of the effective quartic field theory due to a cubic anisotropy, and again suggests a discontinuous phase transition.
\end{abstract}

PACS numbers: 67.85.Hj, 03.75.Mn, 64.60.ae, 75.10.Hk

\section{INTRODUCTION}

The seminal studies [1-3] reported the observation of Bose-Einstein condensation (BEC) in time-of-flight images, which revealed that ultracold bosons accumulate near the minimum of the dispersion relation and condense below a critical temperature. This second order phase transition is the quintessential example for $\mathrm{U}(1)$ symmetry breaking, described by complex $\phi^{4}$ theory. Subsequently, numerous studies have addressed the question if and how BEC can be achieved, by including symmetries apart from the conventional U(1) symmetry $[4,5]$. Populating bosons in spatially anisotropic orbitals of an optical lattice, for instance, has been proposed to create exotic superfluid orders [6-19]. In the experiment reported in $[20,21]$, a long-lived metastable state of ultracold bosons in the $p$-band was indeed realized and displays condensation involving two different momenta, visible in time-of-flight measurements. Both experimental work [22] as well as theoretical analysis $[8,13]$ suggest that the two momentum states form a superposition with an imaginary relative phase, in which the atoms condense, leading to superfluid order with broken timereversal symmetry. By exploiting a matter wave heterodyning technique, the breaking of time-reversal symmetry via the spontaneous formation of chiral order in this $p$-band bipartite optical lattice is revealed without ambiguity [23].

Another method to create unconventional BEC is to load bosons into the Floquet states of a shaken optical

\footnotetext{
*Email:wenmin@phys.nchu.edu.tw

†Email:lmathey@physnet.uni-hamburg.de
}

lattice [24-29]. In such a shaken triangular lattice the effective tunneling parameters can be tuned to negative values [30,31]. The bosonic atoms condense at the minima of the effective dispersion at non-zero momenta. This was used to perform simulations of frustrated classical magnetism in [31]. Furthermore, complex-valued effective tunneling parameters can be created, which correspond to artificial gauge fields [32,33]. These artificial gauge fields couple to an emergent, chiral order parameter, as demonstrated in Ref.[34].

The frustrated bosonic system studied in Ref.[34], as sketched in Fig. 1 (a), is related to a classical XY model in the triangular lattice by ignoring phase fluctuations along the z-axis. The underlying physics is to represent the bosonic fields $\langle\psi(\boldsymbol{r})\rangle=\sqrt{n(\boldsymbol{r})} e^{i \varphi(\boldsymbol{r})}$ in the phasedensity representation, thus the phase $\varphi(\boldsymbol{r})$ is mapped onto a classical XY spin, $\boldsymbol{s}(\boldsymbol{r})=(\cos \varphi(\boldsymbol{r}), \sin \varphi(\boldsymbol{r}))$ [35]. For a negative tunneling parameter $J_{e}<0$ in this approximation results in antiferromagnetic spin coupling between neighboring sites $[34,36]$. This two-dimensional (2D) antiferromagnetic XY model is frustrated due to its triangular geometry, and has two degenerate ground states, discriminated by their chiral order, as illustrated in Fig. 1 (c), [37-39]. Thus, in addition to breaking the $\mathrm{U}(1)$ symmetry of the system, spontaneous breaking of an emergent, chiral $\mathbb{Z}_{2}$ symmetry occurs [38-40].

In this paper, we explore the critical behavior of an interacting Bose gas, realized in Ref.[31, 34, 36]. In the $x y$-plane, as shown in Fig. 1 (a), the bosons move in a triangular optical lattice with a real-valued, the negative effective tunneling parameter $J_{e}<0$, while moving freely along the $z$-direction. We therefore include the three dimensional character of the system, and the density fluctuations. We develop a field theoretical description of the system, which has the form of a two-component Bose 


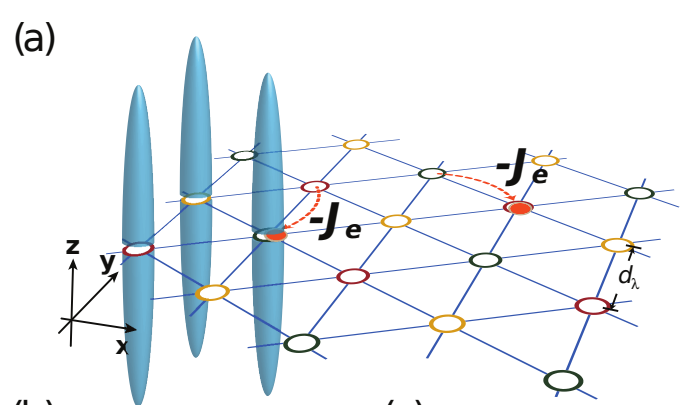

(b)

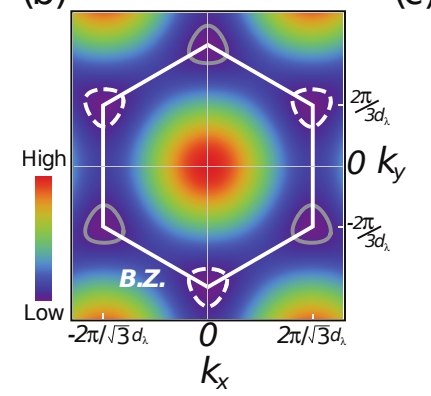

(c)

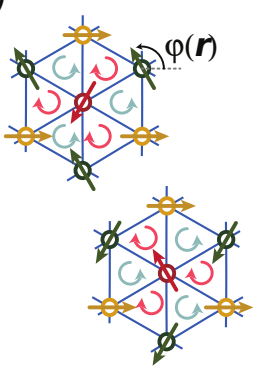

Figure 1: (a) Sketch of the two-dimensional triangular lattice of weakly coupled $1 \mathrm{~d}$ systems. $J_{e}$ is the effective tunneling parameter, with $J_{e}<0$. The bosons move freely in the $z$ direction, and in an optical lattice in the $x-y$ plane. (b) Contour plot of the energy dispersion in the first Brillouin zone (BZ) for the triangular lattice model, in the $k_{x}-k_{y}$ plane, based on Eq. 3. Contours near the two distinct minima are depicted as dashed and solid lines. (c) Phase configurations of the two condensates at momenta $\mathbf{k}_{1}$ and $\mathbf{k}_{2}$ are equivalent to the spin fields $\boldsymbol{s}(\boldsymbol{r})=(\cos \varphi(\boldsymbol{r}), \sin \varphi(\boldsymbol{r}))$.

gas. The two species describe the bosons near the two minima of the dispersion, as shown in Fig. 1 (b). In this description, the $\mathrm{U}(1)$ symmetry is broken if one of the two species is condensed to the nearby minima. Moreover, the $Z_{2}$ symmetry breaking is analogous to condensation at only one of the two degenerate energetic minima $[34,36]$. The weak contact repulsion of the bosons is also decomposed into the interaction within one component, $V_{0}$, and the interaction between these two components, $V_{12}$, in the two-component Bose gas.

By regarding these interactions as two independent parameters in a Huang-Yang-Luttinger (HYL) approach [41], we find that the critical behavior of the $U(1) \times$ $U(1) \times \mathbb{Z}_{2}$ symmetry breaking is controlled by the ratio of these two interactions in the mean-field scheme. The HYL approximation predicts that the $\mathbb{Z}_{2}$ symmetry breaking is a continuous, second-order phase transition for $1<V_{12} / V_{0} \lesssim 1.5$. However, the $\mathbb{Z}_{2}$ symmetry breaking becomes discontinuous, first-order, for $V_{12} / V_{0} \gtrsim 1.5$. For $V_{12} / V_{0}<1$, the two $U(1)$ symmetries are spontaneously broken as the temperature is decreased, while the $\mathbb{Z}_{2}$ symmetry is preserved.

To further study the phase diagram beyond the meanfield scheme, we perform a one-loop renormalization group (RG) calculation, resulting in a flow of the chemi- cal potential and the coupling constants. This calculation clarifies that there are two regimes: For $0<V_{12} / V_{0} \leq 1$, $V_{12}$ and $V_{0}$ flow towards a Heisenberg fixed point, at which $V_{12}=V_{0}$, describing a second order phase transition that differs from the condensation transition of a single-component system. For $V_{12} / V_{0}>1$, however, $V_{0}$ flows towards a negative value, while $V_{12}$ increases and remains positive. This result implies the breakdown of the quartic theory, and higher-order terms, for instance a three-body interaction, need to be included. It also indicates that a discontinuous phase transition occurs [57], due to a cubic anisotropy. Thus, we conclude that the critical behavior in the regime $V_{12} / V_{0}>1$ is of first order, improving on the HYL approximation. We conclude that for the bare interaction of the system, the ratio $V_{12} / V_{0}=2$, suggests that the transition is first order.

This paper is organized as follows: in Sec. II we develop the field theoretic description of the lattice model. In Sec. III we study the free energy based on the HuangYang-Luttinger approximaiton and investigate the phase diagram for different ratios of $V_{12} / V_{0}$. In Sec. IV, we study the critical behavior in the framework of a oneloop RG calculation, and in Sec. V we conclude.

\section{EFFECTIVE FIELD THEORY}

The system, as depicted in Fig. 1 (a), is described by the Hamiltonian $H=H_{0}+H_{I}$, with

$$
\begin{aligned}
H_{0}=\int d z & \left\{\sum_{\boldsymbol{r}} \psi^{\dagger}(\boldsymbol{r}, z)\left(\frac{-\hbar^{2} \partial_{z}^{2}}{2 m_{0}}-\mu_{3 D}\right) \psi(\boldsymbol{r}, z)\right. \\
& \left.+\left|J_{e}\right| \sum_{\left\langle\boldsymbol{r}, \boldsymbol{r}^{\prime}\right\rangle}\left[\psi^{\dagger}(\boldsymbol{r}, z) \psi\left(\boldsymbol{r}^{\prime}, z\right)+\text { h.c. }\right]\right\}
\end{aligned}
$$

and the interaction term

$$
H_{I}=\frac{U}{2} \sum_{\boldsymbol{r}} \int d z \psi^{\dagger}(\boldsymbol{r}, z) \psi^{\dagger}(\boldsymbol{r}, z) \psi(\boldsymbol{r}, z) \psi(\boldsymbol{r}, z) .
$$

$\left\langle\boldsymbol{r}, \boldsymbol{r}^{\prime}\right\rangle$ represents nearest-neighbor pairs of sites and $m_{0}$ is the mass of the atoms. $\mu_{3 D}$ is the chemical potential, and $U$ denotes the magnitude of the repulsive contact interaction. The frustration of the system is due to the negative value of the tunneling parameter $J_{e}$. Neglecting the interaction term and the chemical potential, we obtain the dispersion relation

$$
\begin{aligned}
\varepsilon(\mathbf{k}) & =\left|J_{e}\right|\left[2 \cos \left(d_{\lambda} k_{y}\right)+2 \cos \left(d_{\lambda} \sqrt{3} k_{x} / 2-d_{\lambda} k_{y} / 2\right)\right. \\
& \left.+2 \cos \left(d_{\lambda} \sqrt{3} k_{x} / 2+d_{\lambda} k_{y} / 2\right)\right]+\hbar^{2} k_{z}^{2} /\left(2 m_{0}\right)
\end{aligned}
$$

with the lattice spacing $d_{\lambda}=2 \lambda_{L} / 3$, and $\lambda_{L}$ being the wavelength of the laser creating the $2 \mathrm{D}$ lattice potential [34]. The dispersion is shown in Fig. 1 (b). It displays two energetically degenerate minima, located at the two distinct momenta $\mathbf{k}_{1 / 2}=\left(2 \pi /\left(\sqrt{3} d_{\lambda}\right), \pm 2 \pi /\left(3 d_{\lambda}\right)\right)$, at 
the boundary of the first Brillouin zone. The classical spins $\boldsymbol{s}(\boldsymbol{r})$ depicted in Fig. 1 (c) correspond to the real and imaginary part of the plane waves $\exp \left(i \mathbf{k}_{1} \boldsymbol{r}\right)$ and $\exp \left(i \mathbf{k}_{2} \boldsymbol{r}\right)$.

The hidden $U(1) \times U(1) \times \mathbb{Z}_{2}$ symmetry of the system emerges as follows. At low temperatures, the bosons will accumulate near the minima. If the bosons condense at one of the two minima, one $U(1)$ symmetry is broken due to condensation, and the $\mathbb{Z}_{2}$ symmetry, which corresponds to the density imbalance between the minima. However, if the bosons condense at both minima with equal density, the $\mathbb{Z}_{2}$ symmetry is preserved, while both $U(1)$ symmetries are broken.

We now derive the low-energy field theoretical description. At low temperatures, bosons will be distributed around the minima $\mathbf{k}_{1}$ and $\mathbf{k}_{2}$. Therefore, we decompose the bosonic field in momentum space as

$$
\begin{aligned}
\psi(\boldsymbol{r}, z) & =\frac{1}{\sqrt{N}} \sum_{\boldsymbol{k}} e^{i \boldsymbol{k} \cdot \boldsymbol{r}} \psi(\boldsymbol{k}, z) \\
& \simeq \frac{\mathcal{A}_{\lambda}^{2}}{\sqrt{N}} \sum_{j=1,2} e^{i \boldsymbol{k}_{j} \cdot \boldsymbol{r}} \int_{\left|\boldsymbol{q}_{j}\right|<\Lambda_{q}} \frac{d^{2} \boldsymbol{q}}{4 \pi^{2}} e^{i \boldsymbol{q}_{j} \cdot \boldsymbol{r}} \phi_{j}\left(\boldsymbol{q}_{j}, z\right) \\
& =\mathcal{A}_{\lambda} \sum_{j=1,2} \phi_{j}(\boldsymbol{r}, z) e^{i \boldsymbol{k}_{j} \cdot \boldsymbol{r}},
\end{aligned}
$$

where $N$ is the total number of sites in the xy-plane, and $\boldsymbol{q}_{j}=\boldsymbol{k}-\boldsymbol{k}_{j}$. We define the slowly varying fields via $\phi_{j}(\boldsymbol{r}, z) \equiv \frac{\mathcal{A}_{\lambda}}{\sqrt{N}} \int_{\left|\boldsymbol{q}_{j}\right|<\Lambda_{q}} \frac{d^{2} \boldsymbol{q}}{4 \pi^{2}} e^{i \boldsymbol{q}_{j} \cdot \boldsymbol{r}} \phi_{j}\left(\boldsymbol{q}_{j}, z\right)$ with $\phi_{j}\left(\boldsymbol{q}_{j}, z\right) \equiv \psi_{j}\left(\boldsymbol{k}_{j}+\boldsymbol{q}_{j}, z\right) / \mathcal{A}_{\lambda}$, where $\Lambda_{q}$ is the momentum cutoff, and $\mathcal{A}_{\lambda}=\sqrt{3} d_{\lambda}^{2} / 2$ is the area in the xy-plane that corresponds to a single site, when mapped on a continuum description. By expressing the Hamiltonian of Eq. (1) in terms of the fields $\phi_{j}(\boldsymbol{r}, z)$, we obtain

$$
H_{0}^{\mathrm{eff}}=\int d^{3} \boldsymbol{R} \sum_{j=1,2} \sum_{a} \phi_{j}^{\dagger}(\boldsymbol{r}, z)\left\{-\frac{\hbar^{2} \partial_{a}^{2}}{2 m_{a}}-\mu\right\} \phi_{j}(\boldsymbol{r}, z) .
$$

Here, $a=x, y, z, m_{x}=m_{y}=m_{J}=\hbar^{2} /\left(3 d_{\lambda}^{2}\left|J_{e}\right|\right)$, $\boldsymbol{R}=(\boldsymbol{r}, z)=(x, y, z), m_{z}=m_{0} \simeq 0.021 m_{J}$, and $\mu$ is the chemical potential of the Bose gas in the continuum description. The fields $\phi_{j}(\boldsymbol{r}, z)$ have a quadratic dispersion relation centered at momentum $\left(\boldsymbol{k}_{j}, k_{z}=0\right)$, respectively,

$$
\varepsilon_{\mathrm{eff}}\left(\boldsymbol{q}_{j}, k_{z}\right)=\frac{\hbar^{2} q_{j x}^{2}}{2 m_{J}}+\frac{\hbar^{2} q_{j y}^{2}}{2 m_{J}}+\frac{\hbar^{2} k_{z}^{2}}{2 m_{z}},
$$

with $\boldsymbol{q}_{j}=\left(q_{j x}, q_{j y}\right)=\boldsymbol{k}-\boldsymbol{k}_{j}$ and $j=1,2$. Expressed in terms of $\phi_{j}(\boldsymbol{r}, z)$, the interaction in Eq. (2) is

$$
\begin{aligned}
& H_{I}^{\mathrm{eff}}=\int d^{3} \boldsymbol{R}\left\{\frac{V_{0}}{2} \sum_{j=1,2} \phi_{j}^{\dagger}(\boldsymbol{R}) \phi_{j}^{\dagger}(\boldsymbol{R}) \phi_{j}(\boldsymbol{R}) \phi_{j}(\boldsymbol{R})\right. \\
& \left.+V_{12} \phi_{1}^{\dagger}(\boldsymbol{R}) \phi_{2}^{\dagger}(\boldsymbol{R}) \phi_{2}(\boldsymbol{R}) \phi_{1}(\boldsymbol{R})\right\},
\end{aligned}
$$

where $V_{0}$ is the intra-component interaction and $V_{12}$ is the inter-component one. It is noticed that the effective interaction is forbidden to exchange species because of the momentum conservation of the original Hamiltonian, Eq. (2). Secondly, the bare magnitudes of these parameters are $V_{0}=U / A_{\lambda}$ and $V_{12}=2 U / A_{\lambda}$, so in particular we have $V_{12}=2 V_{0}$. However, under the RG flow that we derive below, these parameters will flow independently. This forces us to regard $V_{0}$ and $V_{12}$ as two independent variables throughout, because their ratio is not protected by a symmetry. Furthermore, our analysis covers the full universality class of this type, not necessarily limited to the original system on a triangular lattice. The total effective Hamiltonian $H^{\text {eff }}=H_{0}^{\text {eff }}+H_{I}^{\text {eff }}$ is a two-component complex $\phi^{4}$-theory, with a single chemical potential $\mu$, because only the total density of the bosons is conserved.

\section{HUANG-YANG-LUTTINGER APPROACH}

As a first insight into the critical behavior of the system, we compute the free energy of the effective Hamiltonian within the Huang-Yang-Luttinger approximation [41]. In this approach, the free energy $A$ is expanded in the interaction strength. Thus, the zero order term $A_{0}$ is the free energy of two ideal Bose gases.

To formulate this quantity, we define the average thermal de Broglie wavelength

$$
\lambda=\left(\lambda_{0} \lambda_{J}^{2}\right)^{1 / 3},
$$

with $\lambda_{0} \equiv 2 \pi \hbar / \sqrt{2 \pi m_{0} k_{B} T}$ and $\lambda_{J} \equiv 2 \pi \hbar / \sqrt{2 \pi m_{J} k_{B} T}$. The density of excited atoms for each of the minima is

$$
n_{e, j}=\frac{1}{\lambda^{3}} \mathcal{G}_{3 / 2}\left(z_{j}\right),
$$

with $j=1,2$, and $\mathcal{G}_{p}=\sum_{l=1}^{\infty} z^{l} / l^{p}$ being the standard Bose function. $z_{j}=\exp \left(\mu_{j} / k_{B} T\right)$ is the fugacity and is computed by the inverse function of Eq. (9) in the free energy below. Here we formally introduce two chemical potentials $\mu_{j}$, but for the result further down we preserve only the total density. The total density of this system is $n_{3 D}=n_{1}+n_{2}$, where $n_{j}$ is the density for the $j$ th component of the system, and is fixed in free energy computation below. The density of the ground states is defined as $n_{0, j}=n_{j}-n_{e, j}$, which leads to a condensate density of $n_{0, j}=n_{j}-\frac{1}{\lambda^{3}} \mathcal{G}_{3 / 2}(1)$, if $n_{j}>\frac{1}{\lambda^{3}} \mathcal{G}_{3 / 2}\left(z_{j}=1\right)$. Therefore, the free energy is given by $A_{0}=A_{0,1}+A_{0,2}$ with

$$
\frac{A_{0, i}}{\mathcal{V}}= \begin{cases}-\frac{k_{B} T}{\hbar^{3}} \mathcal{G}_{5 / 2}\left(z_{i}\right)+n_{i} k_{B} T \ln \left(z_{i}\right) & \text { if } z_{i}<1, \\ -\frac{k_{B} T}{\lambda^{3}} \mathcal{G}_{5 / 2}(1) & \text { if } z_{i}=1,\end{cases}
$$

and $\mathcal{V}$ being the volume of the system.

We now compute the next order of the free energy $A_{I}$ which is due to and linear in the interaction term (7). We approximate $\left\langle\phi_{j}^{\dagger}\left(\boldsymbol{q}_{j}=0, k_{z}=0\right) \phi_{j}\left(\boldsymbol{q}_{j}=0, k_{z}=\right.\right.$ $0)\rangle=n_{0, j}$ and $\left\langle\phi_{j}^{\dagger}\left(\boldsymbol{q}_{j}, k_{z}\right) \phi_{j}\left(\boldsymbol{q}_{j}, k_{z}\right)\right\rangle=n_{j}$, according to 
(a)

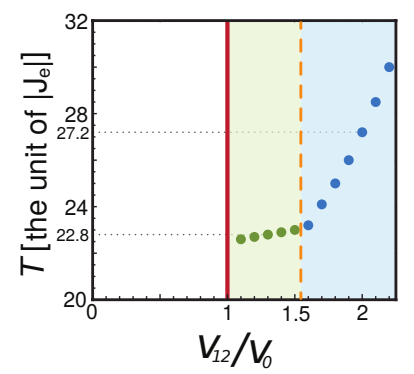

(b) $V_{12}=1.3 V_{0}$

(c) $V_{12}=2 V_{0}$

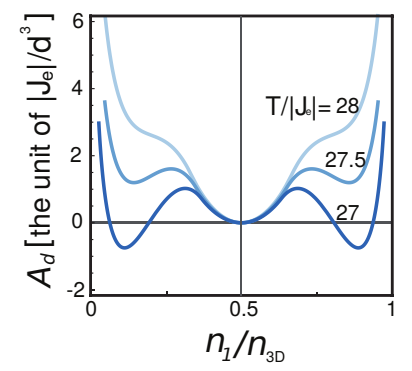

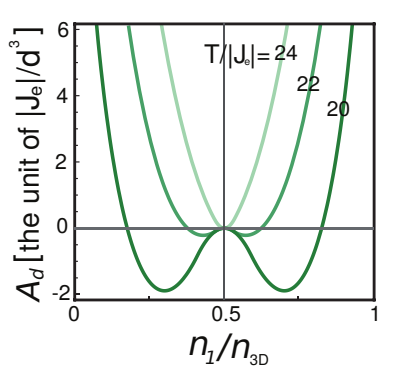

(d) $V_{12}=V_{0}$

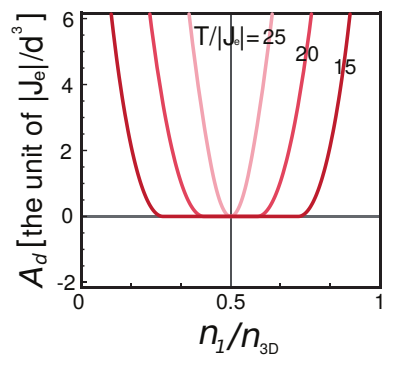

Figure 2: (a) The critical temperature of the system, as a function of $\gamma_{12}=V_{12} / V_{0}$. For $V_{12}<V_{0}$, the transition corresponds to breaking both $U(1)$ symmetries, while the $\mathbb{Z}_{2}$ symmetry is preserved. For $V_{12}>V_{0}$, one of the $U(1)$ symmetries and the $\mathbb{Z}_{2}$ symmetry are broken. The green shaded regime corresponds to a second order transition of $\mathbb{Z}_{2}$ symmetry breaking, the blue-shaded regime to a first order transition. The critical temperatures for $V_{12}=1.3 V_{0}$ and $V_{12}=2 V_{0}$ are 22.8 and 27.2 in the unit of $\left|J_{e}\right|$ respectively. (b) In the regime of $1<\gamma_{12} \lesssim 1.5$, the two minima of $A_{d}\left(n_{1}\right)$ in Eq. (12) evolve away from $n_{1}=n_{3 \mathrm{D}} / 2$ gradually, indicating a continuous phase transition (green lines). The critical temperature for $V_{12}=1.3 V_{0}$ is 22.8 marked in the panel (a) For $1.5 \lesssim \gamma_{12}<2.5$, the minima of $A_{d}\left(n_{1}\right)$ suddenly occur away from the symmetry point $n_{1}=n_{3 \mathrm{D}} / 2$, corresponding to a discontinuous phase transition (blue lines). (d) At $\gamma_{12}=1$, a plateau grows at $A_{d}\left(n_{1}\right)=0$ by decreasing temperature.

the HYL approximation. Therefore, the first order contribution to the free energy is

$$
\frac{A_{I}}{\mathcal{V}}=\frac{V_{0}}{2}\left[2 n_{1}^{2}+2 n_{2}^{2}-n_{0,1}^{2}-n_{0,2}^{2}\right]+V_{12} n_{1} n_{2}
$$

We explore the properties of this approximation of the free energy $A_{0}+A_{I}$ for the experimental parameters of Ref. [34], for various ratios of $V_{12} / V_{0}$ and temperatures, and with fixed total density $n_{3 D}$. In units of the lattice constant $d_{\lambda}=553 \mathrm{~nm}$ and the effective tunneling parameter $\left|J_{e}\right|=k_{B} \times 0.26 \mathrm{nK}$, the interaction strength is given by $V_{0} \simeq 37.78\left|J_{e}\right| d_{\lambda}^{3}$, and we have a density of $n_{3 \mathrm{D}} \simeq 2.88 d_{\lambda}^{-3}$ of ${ }^{87} \mathrm{Rb}$ atoms.

At low temperatures, the density of the two condensates $n_{0, j}$ becomes non-zero, which implies a U(1) symmetry breaking. To analyze the critical behavior of the chiral phase transition, we consider the magnitude of the free energy relative to the $\mathbb{Z}_{2}$-symmetric state with
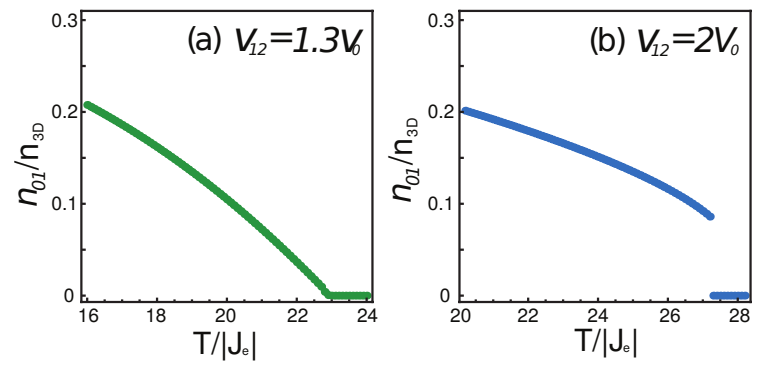

Figure 3: The condensate density $n_{0,1}$ as a function of temperature for (a) $V_{12} / V_{0}=1.3$ and (b) $V_{12} / V_{0}=2$ in the HYL approximation.

$$
\begin{aligned}
& n_{1}=n_{2}=n_{3 D} / 2 \\
& \begin{aligned}
A_{d}\left(n_{1}\right)=\frac{1}{\mathcal{V}}\left[A_{0}\left(n_{1}\right)+A_{I}\left(n_{1}\right)\right. \\
\left.\quad-A_{0}\left(n_{3 D} / 2\right)-A_{I}\left(n_{3 D} / 2\right)\right] .
\end{aligned}
\end{aligned}
$$

If $A_{d}\left(n_{1}\right)$ becomes negative as the temperature is lowered, it indicates that the $\mathbb{Z}_{2}$ symmetry is broken. Furthermore, where the new minima emerge indicates if the phase transition is first or second order.

We find that for $V_{12} / V_{0}<1$ the minimum of $A_{d}\left(n_{1}\right)$ is always located at $n_{1}=n_{3 \mathrm{D}} / 2$, indicating that the $\mathbb{Z}_{2}$ symmetry is preserved. However, for $V_{12} / V_{0}>1$, we find that the $\mathbb{Z}_{2}$ symmetry breaks, as new minima appear away from the symmetric point. The temperature at which these minima occur is the critical temperature of the $\mathbb{Z}_{2}$ symmetry breaking. It is plotted against the ratio $\gamma_{12}=V_{12} / V_{0}$ in Fig. 2 (a). We also find that the order of the $\mathbb{Z}_{2}$ symmetry breaking changes as $V_{12} / V_{0}$ is varied. As shown in Fig. 2 (b), the two minima of $A_{d}\left(n_{1}\right)$ emerge continuously at $n_{1}=n_{3 \mathrm{D}} / 2$, indicating a second order phase transition in the regime of $1<V_{12} / V_{0} \lesssim 1.5$. In this regime, as shown in Fig. 2 (a), the critical temperature of the $\mathbb{Z}_{2}$ symmetry breaking only increases weakly with increasing $V_{12} / V_{0}$. However, for $1.5 \lesssim \gamma_{12}<2.5$, the minima of $A_{d}\left(n_{1}\right)$ emerge away from the symmetric point at $n_{1}=n_{3 \mathrm{D}} / 2$, as demonstrated in Fig. 2 (c), indicating a discontinuous phase transition. The critical temperature rapidly increases with increasing $V_{12} / V_{0}$, as illustrated in Fig. 2 (a). For $V_{12} / V_{0}=1, A_{d}$ develops a plateau at $A_{d}=0$, below the critical temperature, as shown in Fig. 2 (d). This reflects the emergent $S U(2)$ symmetry at this interaction strength, which corresponds to a Heisenberg fixed point, as we discuss below.

We again emphasize that this analysis suggests a first transition of the system, because the bare value of the interaction is $V_{12} / V_{0}=2$. Furthermore, as we discuss in the next section, our RG analysis suggests that the first order regime extends throughout the entire regime $V_{12}>V_{0}$.

We note that the $\mathbb{Z}_{2}$ and the $U(1)$ symmetry breaking occur at the same temperature, within the HYL ap- 
proximation. This occurs because the condensate density is responsible for generating the two minima in the free energy. We demonstrate this computing the condensate fraction $n_{0,1}$ versus temperature in Fig. 3 (a) for $V_{12} / V_{0}=1.3$ and (b) $V_{12} / V_{0}=2$ respectively. The critical temperature of the $U(1)$ symmetry breaking is the same as the temperature at which $n_{0,1}$ becomes nonzero. The first- and second-order phase transitions of the simultaneous $\mathbb{Z}_{2}$ and $U(1)$ symmetry breaking are indicated by the discontinuous and continuous change of the condensate density in Fig 3.

\section{RENORMALIZATION GROUP APPROACH}

\section{A. Renormalization Group flow}

To study the phase diagram systematically, we perform a renormalization group calculation at one-loop order in the weak-coupling regime[42]. We write the partition function as a path integral:

$$
\mathcal{Z}=\int \mathcal{D}\left[\phi^{*}, \phi\right] e^{-S\left[\phi^{*}, \phi\right] / \hbar}
$$

with the action

$$
S\left[\phi^{*}, \phi\right] \equiv \int_{0}^{\hbar \beta} d \tau \sum_{j=1,2}\left[\phi_{j}^{*}\left(\hbar \partial_{\tau}\right) \phi_{j}+H\left(\phi_{j}^{*}, \phi_{j}\right)\right],
$$

and $H=H_{0}^{\mathrm{eff}}+H_{I}^{\mathrm{eff}}$ in the coherent-state representation of the Hamiltonian $H$. We consider the classical limit of the effective action $S\left[\phi^{*}, \phi\right]$, i.e. we ignore the dependence of the fields on the Matsubara time $\tau, \phi_{j}(\tau)=\phi_{j}$. This is equivalent to only taking the $\omega=0$ Matsubara frequency into account [42]. In this approximation, the partition function simplifies to

$$
\mathcal{Z}=\int \mathcal{D}\left[\phi^{*}, \phi\right] e^{-\beta F_{L}\left[\phi^{*}, \phi\right]},
$$

where $F_{L}\left[\phi^{*}, \phi\right]=F_{L, 0}+F_{L, I}$ is the Landau free energy functional with

$$
F_{L, 0}=\int d^{3} \boldsymbol{R} \sum_{j=1,2}\left[\frac{\hbar^{2}}{2 m^{*}}\left|\nabla \phi_{j}\right|^{2}-\mu\left|\phi_{j}\right|^{2}\right],
$$

and the interaction

$$
F_{L, I}=\int d^{3} \boldsymbol{R}\left[\frac{V_{0}}{2}\left(\left|\phi_{1}\right|^{4}+\left|\phi_{2}\right|^{4}\right)+V_{12}\left|\phi_{1}\right|^{2}\left|\phi_{2}\right|^{2}\right]
$$

We rescale the momenta in the $x$ - and $y$-direction by $\sqrt{3 d^{2}\left|J_{e}\right| m_{z} / \hbar^{2}}$ to arrive at a spatially homogeneous expression, with the effective mass $m^{*}=\left(m_{J}^{2} m_{z}\right)^{1 / 3}$ and $m_{x y}=m_{z}=m^{*} \simeq 0.276 m_{J}$. To perform the RG transformation, we introduce an energy cutoff of this system $\varepsilon_{\Lambda}=\hbar^{2} \Lambda^{2} /\left(2 m^{*}\right)$ with the momentum cutoff $\Lambda$, which sets the maximum energy scale of the field theory description. A physical choice for the energy cut-off is the bandwidth of the dispersion in the $\mathrm{x}-\mathrm{y}$ plane, which is determined by $J_{e}$.

We note that in the following we assume that the low-energy regime of the driven system is approximately given by $\exp (-\beta H)$. The assumption is based on the high-frequency lattice shaking in our system, where the lattice shaking frequency is $\omega=2 \pi \times 2.8 \mathrm{kHz}$ and the bare hopping amplitude is $J_{\text {bare }}=4 \times 10^{-3} \mathrm{E}_{\text {rev }}$ i.e. $\hbar \omega / J_{\text {bare }} \sim 200$ [34]. Though from the eigenstate thermalization hypothesis all the Floquet eigenstates are indistinguishable from the infinite-temperature state or a completely random state at long-time limit [43-51], in this study we are interested in the quasi-stationary(or metastable) state during high-frequency driving. Recent theoretical study shows for an isolated Floquet systems in high-frequency driving with local interactions, a nonintegrated system as we considered here, heating will be exponentially slow. It implies that prethermalized Floquet many-body phases, though metastable, will be very long-lived [52]. On the other hand, because the energy is almost conserved up to timescale $e^{\mathcal{O}\left(\hbar \omega / J_{\text {bare }}\right)}$, the system will first reaches a quasi-stationary state with a $f$ nite temperature [53, 54]. Thus, thermal equilibrium due to the approximative character of the Floquet Hamiltonian is well-approximated for the large frequencies and the experimentally relevant times considered. Such that, the ensemble average of the periodically driven isolated systems in the intermediate timescale is approximated by the ensemble average of the quasi-stationary state(or Floquet Hamiltonian) in this study. The above concept is also applied in recent theoretical studies [55, 56].

In an RG step, we split the fields $\phi_{j}$ into a low-energy and a high-energy part. The high-energy degrees of freedom are integrated out to obtain a renormalized free energy functional with renormalized chemical potential $\mu$ and coupling constants $V_{0}$ and $V_{12}$, which we compute at one-loop order. Iterating this RG step results in a set of flow equations, given below. The derivation of the flow equations using the $\varepsilon$-expansion is discussed in Appendix A.

We define the dimensionless variables $\mu_{\Lambda} \equiv \frac{1}{\varepsilon_{\Lambda}} \mu, g_{0} \equiv$ $\frac{\Lambda^{3}}{2 \pi^{2} \varepsilon_{\Lambda}} V_{0}, g_{12} \equiv \frac{\Lambda^{3}}{2 \pi^{2} \varepsilon_{\Lambda}} V_{12}, T_{\Lambda} \equiv \frac{1}{\varepsilon_{\Lambda}} k_{B} T$. We note that by construction all of these dimensionless quantities are small compared to 1 , because they are divided by the high energy cut-off $\varepsilon_{\Lambda}$. For this reason, we terminate the flow if one of these parameters reaches unity, as described below. In terms of these variables, the flow equations to linear order in $\varepsilon=4-d$ are

$$
\begin{aligned}
\frac{d \mu_{\Lambda}}{d l} & =2 \mu_{\Lambda}-2 g_{0} T_{\Lambda}\left(1+\mu_{\Lambda}\right)-g_{12} T_{\Lambda}\left(1+\mu_{\Lambda}\right), \\
\frac{d g_{0}}{d l} & =\varepsilon g_{0}-5 g_{0}^{2} T_{\Lambda}-g_{12}^{2} T_{\Lambda}, \\
\frac{d g_{12}}{d l} & =\varepsilon g_{12}-2 g_{12}^{2} T_{\Lambda}-4 g_{0} g_{12} T_{\Lambda} .
\end{aligned}
$$

where $l=\ln \left(\Lambda / \Lambda_{b}\right)$ is the logarithm of the ratio between 


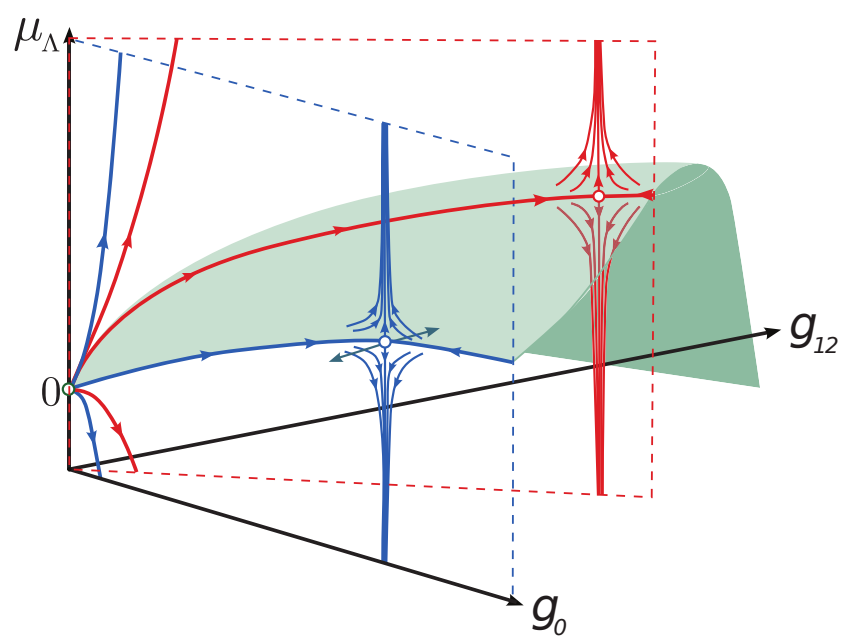

Figure 4: Qualitative sketch of the flow diagram in the space spanned by $g_{0}, g_{12}$ and $\mu_{\Lambda}$. We illustrate the $g_{12}=0$ plane (blue) and the $g_{0}=g_{12}$ plane (red) with flow trajectories in the vicinity of the three fixed points. The boundary of the thermal and condensate phase is sketched as a transparent green surface.

the initial momentum cutoff $\Lambda$ and the running cutoff $\Lambda_{b}$. We note that by setting $g_{12}=0$, these flow equations reduce to the two standard RG equations of the onecomponent, complex $\phi^{4}$ theory.

To identify the fixed points of the flow, we set the Eqs. (18), (19) and (20) to zero. This results in three fixed points, which are given by

$$
\begin{aligned}
\mu_{\Lambda}^{*} & =0, \quad g_{0}^{*}=0, \quad g_{12}^{*}=0, \\
\mu_{\Lambda}^{*} & =\frac{\varepsilon}{5}, \quad g_{0}^{*}=\frac{\varepsilon}{5 T_{\Lambda}}, \quad g_{12}^{*}=0, \\
\mu_{\Lambda}^{*} & =\frac{\varepsilon}{4}, \quad g_{0}^{*}=\frac{\varepsilon}{6 T_{\Lambda}}, \quad g_{12}^{*}=\frac{\varepsilon}{6 T_{\Lambda}},
\end{aligned}
$$

to linear order in $\varepsilon$. For these points, which are illustrated in Fig. 4, the variables remain unchanged under the RG flow. We investigate the flow further by expanding the flow equations to first order around the fixed points (see Appendix B). This expansion shows that the non-interacting fixed point (21) is unstable in all directions. In the vicinity of the second fixed point (22), we recover the flow in the $g_{12}=0$ plane [42], which includes one relevant and one irrelevant direction. In addition we find one more relevant direction, which drives the system away from the $g_{12}=0$ plane. In Fig. 4, we qualitatively sketch the flow diagram in the vicinity of three fixed points. The irrelevant and relevant directions obtained in the fixed-point analysis correspond to the flow toward the fixed points and away from it, respectively. On the $g_{12}=0$ plane, the line through $\left(\mu_{\Lambda}, g_{0}\right)=(0,0)$ and $\left(\varepsilon / 5, \varepsilon /\left(5 T_{\Lambda}\right)\right)$ corresponds to the boundary between the thermal phase and the condensate phase. Below and above this line, the chemical potential flows to large negative and positive values, corresponding to $U(1)$ symmetry preserving and breaking, respectively. For non-zero

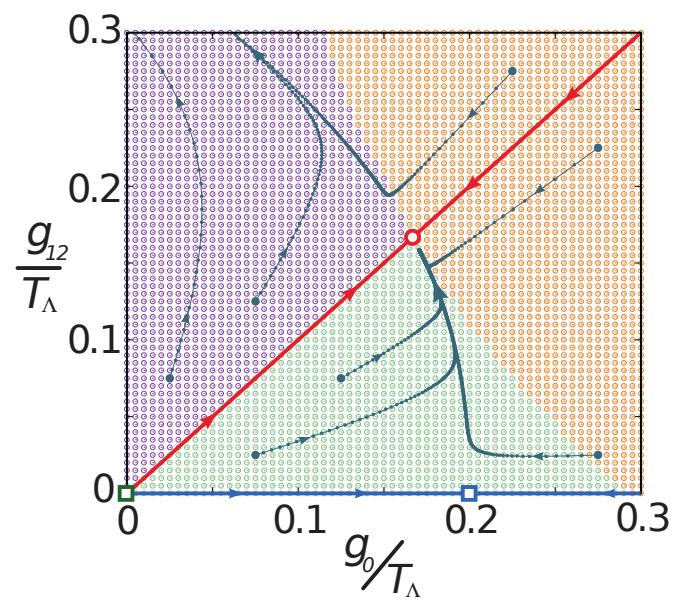

Figure 5: The dark blue lines are flow trajectories projected on the $g_{0}-g_{12}$ plane, obtained by numerical integration of (1820). The empty circles and squares are the locations of the three fixed points projected on the plane. The initial values of the trajectories are shown as blue dots. The initial value of $\mu_{\Lambda}$ is $\mu_{\Lambda}(0)=1 / 3$. $\mu_{\Lambda}$ flows to positive (green and purple circles) values, which indicates a condensed phase, or to negative values (orange circles), indicating a thermal phase. Furthermore, the regime of a discontinuous transition is indicated with purple circles, and the regime of a continuous transition with green circles.

values of $g_{12}$, we find that $g_{12}$ increases under the $\mathrm{RG}$ transformation. This indicates that the inter-component interaction is always relevant in a two-component Bose gas.

The flow behavior in the vicinity of the third fixed point (23) is also depicted in Fig. 4. We refer to this fixed point as a Heisenberg fixed point, because it displays $S U(2)$ symmetry. The fixed-point analysis shows that there is one irrelevant direction along the $g_{0}=g_{12}$ plane, which is similar to the behavior close to the second fixed point on the $g_{12}=0$ plane. In addition, there is a relevant direction, that drives the chemical potential to $\pm \infty$. The line through $\left(\mu_{\Lambda}, g_{0}, g_{12}\right)=(0,0,0)$ and $\left(\varepsilon / 4, \varepsilon /\left(6 T_{\Lambda}\right), \varepsilon /\left(6 T_{\Lambda}\right)\right)$ on the $g_{0}=g_{12}$ plane is the boundary of the transition from the thermal to the condensed phase. This line is part of the critical surface, illustrated as the transparent green plane in Fig. 4, below which the chemical potential flows to $-\infty$, and above which it flows to $+\infty$. We note that this surface bends down to negative values of $\mu$ and $g_{0}$, which is a prerequisite for the first order transition for $g_{12}>g_{0}$, discussed below.

Finally, the flow behavior perpendicular to the $g_{0}=g_{12}$ plane shows marginal behavior in the first-order analysis near the third fixed point, see Appendix B. We integrate the RG equations to study the RG flow further. In Fig. 5, we depict the RG flow in the $g_{0}-g_{12}$ plane, that is created by the Eqs. (18), (19) and (20). As an example, we fixed the initial value of the chemical potential to $\mu_{\Lambda}(0)=1 / 3$. 
We choose several initial values of $\left(g_{0}, g_{12}\right)$ to depict the trajectories, to illustrate the flow.

We also indicate the three fixed points, as well as whether the chemical potential flows to $+\infty$, or towards $-\infty$, via color coding: The orange colored region corresponds to the $\mu \rightarrow-\infty$ regime, whereas the green and purple regions indicate $\mu \rightarrow+\infty$. The latter regime is further divided into two subregimes, which are distinguished by the asymptotic behavior of $g_{0}$ and $g_{12}$ under the flow. If the flow approaches the fixed point $\left(g_{0}^{*}, g_{12}^{*}\right)=$ $\left(\varepsilon /\left(6 T_{\Lambda}\right), \varepsilon /\left(6 T_{\Lambda}\right)\right)$ asymptotically, the initial point is colored green. This occurs for $0<g_{12}(0) / g_{0}(0) \leq 1$. If the flow is repelled by this fixed point, and $g_{0}$ asymptotically flows to a negative value, the initial point is colored purple. This occurs for $g_{12}(0) / g_{0}(0)>1$.

For the regime $0<g_{12}(0) / g_{0}(0) \leq 1$, the critical behavior is controlled by the fixed point at $\left(g_{0}^{*}, g_{12}^{*}\right)=$ $\left(\varepsilon /\left(6 T_{\Lambda}\right), \varepsilon /\left(6 T_{\Lambda}\right)\right)$. The flow towards to this point separates into an initial, fast flow onto the marginal surface, connecting the second and third fixed point, on which it flows logarithmically slow, when parametrized by $l$. The chemical potential on the other hand diverges exponentially fast to either $\pm \infty$. When its absolute value reaches unity, the flow moves out of its range of validity, and we terminate it. Therefore, the RG flow, while asymptotically moving towards the Heisenberg fixed point, predicts a physical state for which $g_{12}^{*}<g_{0}^{*}$. This suggests that the system breaks both $U(1)$ symmetries, while the $\mathbb{Z}_{2}$ is preserved. The perturbation $\sim\left(g_{0}-g_{12}\right)\left(\left|\phi_{1}\right|^{2}-\left|\phi_{2}\right|^{2}\right)^{2}$ is therefore dangerously irrelevant: It modifies the ground state of the system in a qualitative way from the state that it would have if the magnitude of $g_{0}-g_{12}$ was identically zero. The second order phase boundary between this phase with two condensates, each breaking a $U(1)$ symmetry, and the thermal phase, is indicated by the green and orange circles. This phase boundary shifts to larger values of $g_{0}$ and $g_{12}$, when the initial value of $\mu_{\Lambda}$ is increased. When it is reduced, the boundary shifts to smaller values. This indicates that the condensation transition can tolerate larger repulsive interactions, when the chemical potential is larger.

For $g_{12}(0) / g_{0}(0)>1$, the parameters are initially attracted to a line that is approximately perpendicular to the line $g_{0}=g_{12}$, as visible in Fig. 5. Once in the vicinity of this line, the parameters are now repelled by the Heisenberg fixed point. In particular, $g_{0}$ is renormalized to a negative value, while $g_{12}$ increases and remains positive. This indicates the breakdown of the quartic, effective field theory, as the energy of the system is no longer bounded from below. Higher order terms, such as a $\phi^{6}$ term:

$$
F_{6} \sim g_{6} \int d \mathbf{R}\left(\left|\phi_{1}\right|^{6}+\left|\phi_{2}\right|^{6}\right)
$$

have to be included to provide a stable description of the system. Furthermore, it indicates that a first-order phase transition can occur, Ref. [57]. We note that this $\phi^{6}$ term is an effective three-body interaction. It is generated by

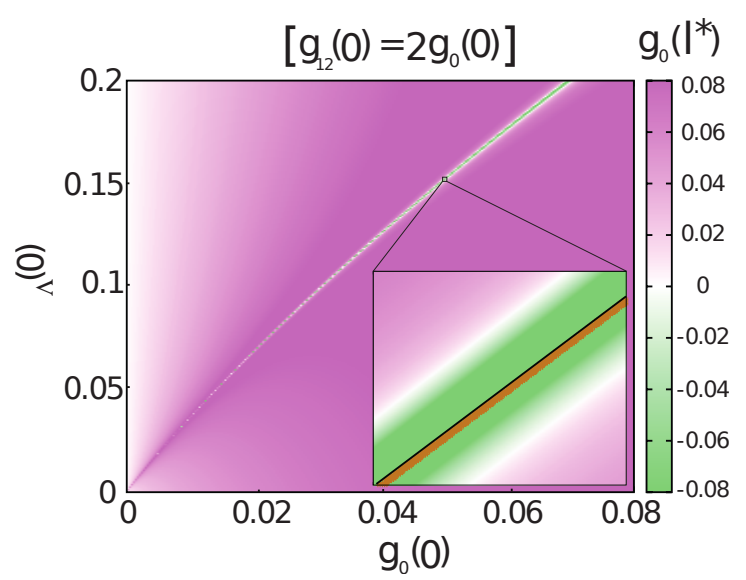

Figure 6: We depict $g_{0}\left(\ell^{*}\right)$ as a function of $g_{0}(0)$ and the chemical potential. Near the critical surface, $g_{0}$ is renormalized to a negative value. In the inset we show the direct vicinity of the critical surface, and also indicate the regime for which $g_{0}^{2} /|\mu|<0.1$, as orange. This regime which exists below the critical surface for any initial value, is responsible for the first order transition of the system.

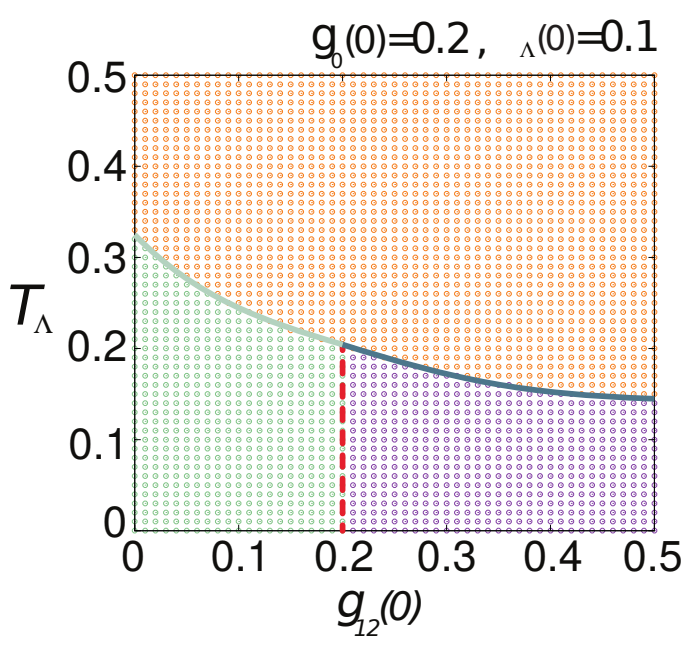

Figure 7: Phase diagram of the system as a function of $g_{12}(0)$ and $T_{\Lambda}$, with fixed $g_{0}(0)=0.2$ and $\mu_{\Lambda}(0)=0.1$. The orange circles denoted the thermal gas phase. The green circles represent the state of two condensates, each breaking a $U(1)$ symmetry, and the purple circles represent a state with a broken $U(1)$ symmetry and a broken chiral, $\mathbb{Z}_{2}$ symmetry. The purple (green) line represents the discontinuous (continuous) phase transition from the thermal gas phase.

integrating out high-energy excitations, and results from virtual two-body collisions. For a deep optical lattice, this has been discussed in Refs. [58, 59]. This scenario is demonstrated in Fig. 6. For a first order transition to occur in this system, an effective free energy with three distinct minima has to emerge, similar to the example in Fig. 2 (c ). For this to occur, the chemical potential has to flow to a negative value, as demonstrated above, 
$g_{0}$ has to flow to a negative value, as shown above, and finally the magnitude of $g_{0}, \mu$ and $g_{6}$ has to be such that three minima occur, and the side minima emerge as the global minima. As can be checked, for this $g_{0}^{2} /|\mu| \gtrsim g_{6}$ is required.

In Fig. 6 we show the $\mu-g_{0}$ plane, where we set $g_{12}=2 g_{0}$, which is the plane relevant to the triangular lattice system. We depict the magnitude of $g_{0}$ for $\ell^{*}$, for which $\left|\mu\left(\ell^{*}\right)\right|=1$. Near the critical surface, $g_{0}$ is negative for any initial value. Furthermore, we depict the regime for which $g_{0}^{2} /|\mu| \gtrsim 0.1$. This, and any other choice for $g_{6}$, results in a narrow regime below the entire critical surface. It is this scenario that suggests a first order transition. We note that a bosonic system of constant density has a monotonously increasing chemical potential, as the temperature is lowered. When the chemical potential reaches the critical surface, the system condenses. For the system of two bosonic fields, and for $g_{12}>g_{0}$, however, before the chemical reaches the critical surface, the effective action develops side minima, which results in a first order transition.

\section{B. Phase diagram}

In Figs. 7 and 8 we show the resulting phase diagram of the system. In Fig. 7 we keep the chemical potential and $g_{0}$ fixed, and vary $g_{12}$ and the temperature. For high temperatures, the system is in the thermal gas phase, of any value of $g_{12}$. This regime is labelled as orange circles. As the temperature is lowered, the system condenses, either into a phase of two condensates, each breaking a $U(1)$ symmetry, or into a chiral condensate phase, which breaks a $U(1)$ and the $\mathbb{Z}_{2}$ symmetry. This transition occurs at $g_{12}(0)=g_{0}(0)$, as illustrated as the red dashed line. Furthermore, the order of the phase transition changes, as $g_{12}$ is increased. For $g_{12}(0)<g_{0}(0)$ the system undergoes a second order phase transition, for $g_{12}(0)>g_{0}(0)$ a first order transition. We also note that the critical temperature is reduced with increasing $g_{12}(0)$. To illustrate the influence of the inter-component interactions $g_{12}(0)$ on the critical temperature further, we show the phase diagram in the $T_{\Lambda}-\mu_{\Lambda}(0)$ plane in Fig. 8. We note that the phase diagram for the interaction found in the experiment [34] is illustrated versus temperature and chemical potential in Fig. 8(c).

\section{CONCLUSIONS}

In this article, we have studied the critical behavior of Bose-Einstein condensation in a frustrated, triangular lattice. Using a renormalization group approach, we have demonstrated that the phase transition is of first order. At this transition, the system breaks both a $U(1)$ symmetry and a $\mathbb{Z}_{2}$, simultaneously, resulting in a condensation with chiral order. We achieve this insight by mapping the system onto a complex $\phi^{4}$ theory consisting of two
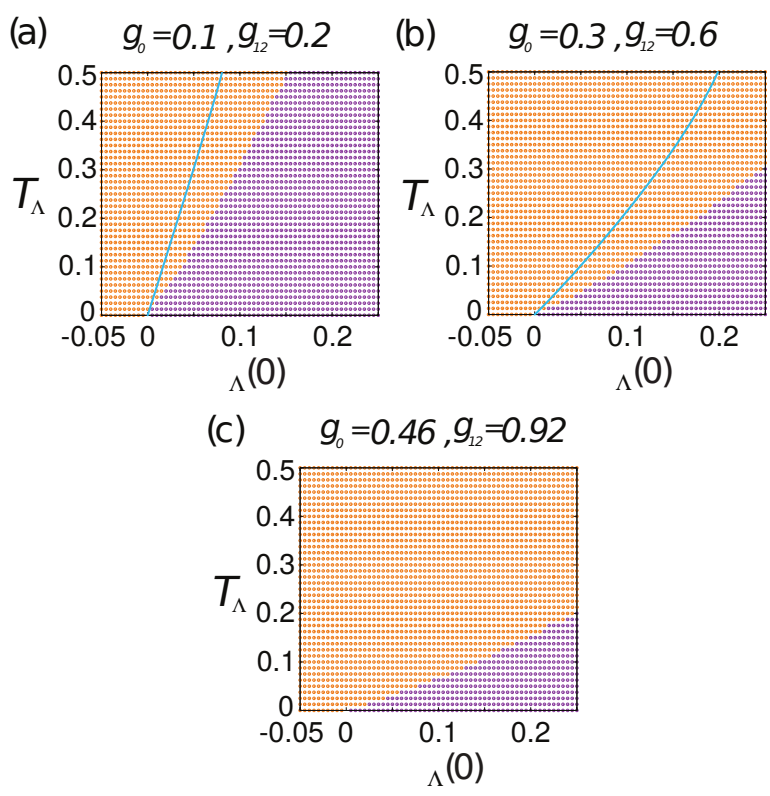

Figure 8: Phase diagrams as a function of $T_{\Lambda}$ and $\mu_{\Lambda}(0)$, with (a) $g_{0}(0)=0.1, g_{12}(0)=0.2$, (b) $g_{0}(0)=0.3, g_{12}(0)=0.6$ and $(\mathrm{c}) g_{0}(0)=0.46, g_{12}(0)=0.92$ (the interaction found in the experiment [34]). Orange circles again label the thermal phase, the condensed phase is depicted with purple circles. To illustrate the renormalization of the critical temperature by $g_{12}$, we depict the blue line, which represents the phase boundary for $(\mathrm{a}) g_{0}(0)=0.1, g_{12}(0)=0$ and (b) $g_{0}(0)=0.3$, $g_{12}(0)=0$.

complex fields. This field theory has a $U(1) \times U(1) \times \mathbb{Z}_{2}$ symmetry, corresponding to the $U(1)$ symmetry of each complex field, as well as a $\mathbb{Z}_{2}$ symmetry of exchanging the two fields. We demonstrate that the critical behavior of this effective field theory is controlled by the ratio of the inter-component $V_{12}$ and the intra-component $V_{0}$ interaction strength. For $V_{12}<V_{0}$ the system undergoes a second order phase transition, in which both $U(1)$ symmetries are broken, while preserving the $\mathbb{Z}_{2}$ symmetry. For $V_{12}>V_{0}$ the system undergoes a first transition, in which one $U(1)$ symmetry and one $\mathbb{Z}_{2}$ symmetry are broken. The latter scenario is reflected in the renormalization group as follows: Rather than being attracted by a fixed point of the flow, which controls the critical behavior, the parameters of the system are repelled by a fixed point, and the emergent free energy becomes unbounded from below. By considering the properties of this flow, this instability can be identified with a first order transition, because the parameters are renormalized in such a way that additional side minima can appear, as in a $\phi^{6}$ theory, and eventually become the global minima. Experimentally, the first order character of the transition could be observed by measuring the condensate fraction and the chiral magnetization as a function of temperature. This intriguing critical behavior, in which condensation occurs as a first order transition, could also occur in numerous other cold atom systems, in which the dis- 
persion of the system has more than one minimum. Our study would apply in an analogous manner, and would therefore be of crucial guidance to understand the critical behavior in such systems. In the end, we note that our analysis might be motivated primarily by the Sengstock experiment, but that this type of analysis similarly applies to other frustraed systems [20].

\section{Acknowledgments}

We acknowledge support from the Deutsche Forschungsgemeinschaft through the SFB 925 and the Hamburg Centre for Ultrafast Imaging, and from the Landesexzellenzinitiative Hamburg, which is supported by the Joachim Herz Stiftung. WMH acknowledges support from the Ministry of Science and Technology in Taiwan through grant MOST104-2112-M-005-006-MY3.

\section{Appendix A: Derivation of the flow equations}

In this Appendix, we derive the renormalization group equations for the free energy functional of interest $(16,17)$. For reasons of clarity, we rewrite the free energy $F_{L}\left[\phi^{*}, \phi\right]=F_{L, 0}+F_{L, I}$ according to:

$F_{L, 0}=\int d^{3} \boldsymbol{R} \sum_{j=1,2}\left[\frac{\hbar^{2}}{2 m^{*}}\left|\nabla \phi_{j}\right|^{2}-\mu_{j}\left|\phi_{j}\right|^{2}\right]$,

$F_{L, I}=\int d^{3} \mathbf{R}\left(\frac{V_{0,1}}{2}\left|\phi_{1}\right|^{4}+\frac{V_{0,2}}{2}\left|\phi_{2}\right|^{4}+V_{12}\left|\phi_{1}\right|^{2}\left|\phi_{2}\right|^{2}\right)$.

This will make it easier to identify distinct terms in the perturbative expansion later on. At the end of the calculation, we will set $\mu_{1}=\mu_{2}=\mu$ and $V_{0,1}=V_{0,2}=V_{0}$. Pictorially, we can represent the two order parameters $\phi_{1}$ and $\phi_{2}$ as colored lines, where the propagator corresponds to the respective chemical potentials and the coupling constants appear as the elementary vertices of the theory (Fig. 9). Assuming the interactions to be weak, the corrections to the propagators and vertices can be obtained using the cumulant expansion of the renormalized free energy functional [42,61].

The first-order correction is given by

$$
\left\langle F_{L, I}\right\rangle_{0,>},
$$

where the expectation value is defined as

$$
\begin{gathered}
\langle\cdots\rangle_{0,>} \equiv \frac{1}{Z_{1,>} Z_{2,>}} \times \\
\iint d\left[\phi_{1,>}^{*}\right] d\left[\phi_{1,>}\right] d\left[\phi_{2,>}^{*}\right] d\left[\phi_{2,>}\right](\cdots) \times \\
e^{-\beta \int d^{3} \mathbf{R}\left(\frac{\hbar^{2}}{2 m_{1}}\left|\nabla \phi_{1,>}\right|^{2}-\mu_{1}\left|\phi_{1,>}\right|^{2}\right)} \times \\
e^{-\beta \int d^{3} \mathbf{R}\left(\frac{\hbar^{2}}{2 m_{2}}\left|\nabla \phi_{2,>}\right|^{2}-\mu_{2}\left|\phi_{2,>}\right|^{2}\right)}
\end{gathered}
$$

with normalization constants $Z_{1,>}$ and $Z_{2,>}$.

To compute the first-order corrections, we split the two complex order parameters into a high-energy and a lowenergy part

$$
\phi_{i}=\phi_{i,<}+\phi_{i,>} \quad \text { with } i=1,2,
$$

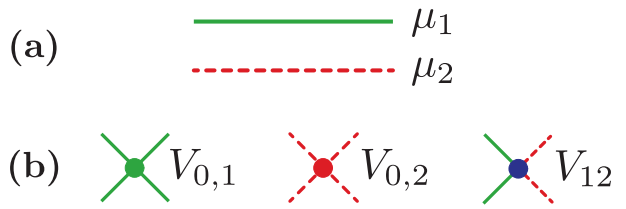

Figure 9: (a) we illustrate the propagators of the first and second components as the green solid and red dashed lines respectively, and (b) corresponding elementary vertices of the theory. 
where:

$$
\begin{aligned}
\phi_{i,<} & =\sum_{k<\Lambda / b} \phi_{i, \mathbf{k}} \frac{e^{i \mathbf{k R}}}{\sqrt{\mathcal{V}}} \quad \text { with } i=1,2, \\
\phi_{i,>} & =\sum_{\Lambda / b<k<\Lambda} \phi_{i, \mathbf{k}} \frac{e^{i \mathbf{k R}}}{\sqrt{\mathcal{V}}} \quad \text { with } i=1,2 .
\end{aligned}
$$

Here, $\mathcal{V}$ is the volume of the system, $\Lambda$ is the high-energy cutoff of the theory and $b$ is some number bigger than one, such that the new cutoff after one RG step is lowered compared to the old one. $\Lambda_{b}=\Lambda / b$ is also referred to as the running cutoff. As can be seen from (A4), the Fourier components of the order parameters that correspond to high-energy excitations are integrated out to obtain corrections for the renormalized free energy functional.

For each of the three addends in (A3), the splitting of the order parameter results in 16 terms. Most terms vanish because of the Gaussian integration in (A4), since they involve an odd number of high-energy parts $\phi_{i,>}$ of the order parameter. Also, $\phi_{i,>}$ must always appear in combination with its complex conjugate to yield a nonzero contribution. Of the remaining terms, only those are of interest that contribute corrections to the chemical potentials (in contrast to just shifting the renormalized free energy functional by a constant factor). Therefore, the only relevant terms for the flow equations are:

$$
\begin{gathered}
4 \frac{V_{0,1}}{2} \int d^{3} \mathbf{R} \phi_{1,<} \phi_{1,<}^{*}\left\langle\phi_{1,>}^{*} \phi_{1,>}\right\rangle_{0,>}, \\
4 \frac{V_{0,2}}{2} \int d^{3} \mathbf{R} \phi_{2,<} \phi_{2,<}^{*}\left\langle\phi_{2,>}^{*} \phi_{2,>}\right\rangle_{0,>}, \\
V_{12} \int d^{3} \mathbf{R} \phi_{2,<} \phi_{2,<}^{*}\left\langle\phi_{1,>}^{*} \phi_{1,>}\right\rangle_{0,>}, \\
V_{12} \int d^{3} \mathbf{R} \phi_{1,<} \phi_{1,<}^{*}\left\langle\phi_{2,>}^{*} \phi_{2,>}\right\rangle_{0,>} .
\end{gathered}
$$

We can see that the $V_{12}$-interaction mixes in corrections from $\mu_{1}$ to $\mu_{2}$ and vice versa. The remaining evaluation of the expectation value can be carried out in Fourier space straightforwardly

$$
\begin{aligned}
\left\langle\phi_{i,>}^{*} \phi_{i,>}\right\rangle_{0,>} & =\frac{1}{\mathcal{V}} \sum_{\Lambda / b<k<\Lambda} \frac{1}{\beta\left(\varepsilon_{i, \mathbf{k}}-\mu_{i}\right)} \\
& =\int_{\Lambda / b}^{\Lambda} \frac{d \mathbf{k}}{(2 \pi)^{d}} \frac{k_{B} T}{\varepsilon_{i, \mathbf{k}}-\mu_{i}},
\end{aligned}
$$

where, in the last step, the continuum limit of the Fourier sum is taken in $d$ dimensions and $\varepsilon_{i, \mathbf{k}}=\hbar^{2} \mathbf{k}^{2} /\left(2 m_{i}\right)$. To mimic our original system, a rescaling of the Fourier components $k \rightarrow k / b$ must be performed after one RG step. The shell in Fourier space that is integrated out is taken to be infinitesimally thin with thickness $d \Lambda=\Lambda d l$ and area $2 \pi^{d / 2} \Lambda^{d-1} / \Gamma\left(\frac{d}{2}\right)$, where $l=\ln (b)$ and $\Gamma(\cdots)$ is the Gamma function. With this, the change in $\mu_{1}$ and $\mu_{2}$ after one RG step caused by the corrections (A8-A11) (a)

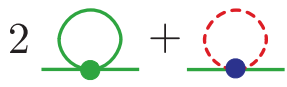

(b)

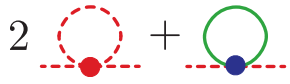

Figure 10: The first-order corrections of the RG method to (a) $\mu_{1}$ and (b) $\mu_{2}$.

can be expressed as

$$
\begin{aligned}
d \mu_{i}= & -2 V_{0, i} \frac{\Lambda^{d}}{(2 \pi)^{d}} \frac{2 \pi^{d / 2}}{\Gamma\left(\frac{d}{2}\right)} \frac{k_{B} T}{e^{-2 l} \varepsilon_{i, \Lambda}-\mu_{i}} e^{-l d} d l \\
& -V_{12} \frac{\Lambda^{d}}{(2 \pi)^{d}} \frac{2 \pi^{d / 2}}{\Gamma\left(\frac{d}{2}\right)} \frac{k_{B} T}{e^{-2 l} \varepsilon_{\bar{i}, \Lambda}-\mu_{\bar{i}}} e^{-l d} d l,
\end{aligned}
$$

where, as before, $i$ is either one or two and $\bar{i}$ is the negation of $i$. These first-order corrections are depicted in Fig. 10. To obtain the desired flow equations, we have to rescale the chemical potentials and the coupling constants according to:

$$
\begin{aligned}
\mu_{i} & \rightarrow \mu_{i} e^{-2 l}, \\
V_{0, i} & \rightarrow V_{0, i} e^{-(4-d) l}, \\
V_{12} & \rightarrow V_{12} e^{-(4-d) l} .
\end{aligned}
$$

The flow equations for $\mu_{1}$ and $\mu_{2}$ take the form:

$$
\begin{aligned}
\frac{d \mu_{i}}{d l}=2 \mu_{i} & -2 V_{0, i} \frac{\Lambda^{d}}{(2 \pi)^{d}} \frac{2 \pi^{\frac{d}{2}}}{\Gamma\left(\frac{d}{2}\right)} \frac{k_{B} T}{\varepsilon_{i, \Lambda}-\mu_{i}} \\
& -V_{12} \frac{\Lambda^{d}}{(2 \pi)^{d}} \frac{2 \pi^{\frac{d}{2}}}{\Gamma\left(\frac{d}{2}\right)} \frac{k_{B} T}{\varepsilon_{\bar{i}, \Lambda}-\mu_{\bar{i}}} .
\end{aligned}
$$

Setting $\mu_{1}=\mu_{2}=\mu$ and $V_{0,1}=V_{0,2}=V_{0}$ results in the $\mathrm{RG}$ equation for the chemical potential.

Now we turn to the second-order correction within the cumulant expansion, which is given by:

$$
\frac{1}{2}\left(\left\langle\left(F_{L, I}\right)^{2}\right\rangle_{0,>}-\left\langle F_{L, I}\right\rangle_{0,>}^{2}\right) .
$$

As usual, corrections to $V_{0,1}, V_{0,2}$ and $V_{12}$ will take the form of connected diagrams. Disconnected diagrams are killed off by the second addend in (A16). We can group the resulting terms into those being proportional to either $V_{0,1}^{2}, V_{0,2}^{2}, V_{12}^{2}, V_{0,1} V_{0,2}, V_{0,1} V_{12}$ or $V_{0,2} V_{12}$. In principle, for each of these six groups, 256 terms must be considered. As in the calculation for the first-order correction, the analysis simplifies, since terms with an odd number of high-energy parts $\phi_{i,>}$ of the order parameter vanish. Also, corrections to the coupling constants should have two incoming and two outgoing low-energy parts $\phi_{i,<}$. Terms with one or three incoming or outgoing $\phi_{i,<}$ factors do not contribute.

Some remaining non-vanishing terms require additional discussion. Terms with only two $\phi_{i,<}$ factors 
would yield further corrections to the chemical potentials. These corrections are of higher order in the coupling constants than the first-order corrections already considered and therefore can be neglected. Terms with $\operatorname{six} \phi_{i,<}$ factors imply an interaction proportional to $\phi^{6}$ not present in the original free energy functional. These terms are usually disregarded in the framework of the $\varepsilon$-expansion [61]. As before, constant shifts to the free energy functional are not of interest.

Strictly, the $\varepsilon$-expansion is only valid near four dimensions. Nevertheless it proved to be successful in lower dimensions as well, especially in the important three-dimensional case. Therefore we are going to use the results obtained using the $\varepsilon$-expansion for a threedimensional system in the main text.

The terms that are relevant for the flow equations will contain an average taken over four fields:

$$
\left\langle\phi_{i,>}^{*} \phi_{i,>} \phi_{j,>}^{*} \phi_{j,>}\right\rangle_{0,>}
$$

Because of the Gaussian integration, this expression is split into products of averages of two fields. When $i=j$, there are two possibilities to contract the fields and a factor of 2 must be introduced.

Now we collect the interesting terms group by group. The two groups proportional to $V_{0, i}^{2}$ contribute the following connected diagrams:

$$
\begin{aligned}
& 16 \frac{V_{0, i}^{2}}{4} \iint d^{3} \mathbf{R} d^{3} \mathbf{R}^{\prime} \phi_{i,<}(\mathbf{R}) \phi_{i,<}^{*}(\mathbf{R}) \phi_{i,<}\left(\mathbf{R}^{\prime}\right) \phi_{i,<}^{*}\left(\mathbf{R}^{\prime}\right) \times \\
&\left\langle\phi_{i,>}^{*}(\mathbf{R}) \phi_{i,>}\left(\mathbf{R}^{\prime}\right)\right\rangle_{0,>}\left\langle\phi_{i,>}^{*}\left(\mathbf{R}^{\prime}\right) \phi_{i,>}(\mathbf{R})\right\rangle_{0,>},
\end{aligned}
$$

$$
\begin{gathered}
4 \frac{V_{0, i}^{2}}{4} \iint d^{3} \mathbf{R} d^{3} \mathbf{R}^{\prime} \phi_{i,<}(\mathbf{R}) \phi_{i,<}^{*}\left(\mathbf{R}^{\prime}\right) \phi_{i,<}(\mathbf{R}) \phi_{i,<}^{*}\left(\mathbf{R}^{\prime}\right) \times \\
\left\langle\phi_{i,>}^{*}(\mathbf{R}) \phi_{i,>}\left(\mathbf{R}^{\prime}\right)\right\rangle_{0,>}\left\langle\phi_{i,>}^{*}(\mathbf{R}) \phi_{i,>}\left(\mathbf{R}^{\prime}\right)\right\rangle_{0,>} .
\end{gathered}
$$

The group of terms proportional to $V_{12}^{2}$ results in a variety of connected diagrams:

$$
\begin{aligned}
V_{12}^{2} \iint d^{3} \mathbf{R} d^{3} \mathbf{R}^{\prime} \phi_{2,<}(\mathbf{R}) \phi_{2,<}^{*}(\mathbf{R}) \phi_{2,<}\left(\mathbf{R}^{\prime}\right) \phi_{2,<}^{*}\left(\mathbf{R}^{\prime}\right) \times \\
\left\langle\phi_{1,>}^{*}(\mathbf{R}) \phi_{1,>}\left(\mathbf{R}^{\prime}\right)\right\rangle_{0,>}\left\langle\phi_{1,>}^{*}\left(\mathbf{R}^{\prime}\right) \phi_{1,>}(\mathbf{R})\right\rangle_{0,>}, \\
V_{12}^{2} \iint d^{3} \mathbf{R} d^{3} \mathbf{R}^{\prime} \phi_{1,<}(\mathbf{R}) \phi_{1,<}^{*}(\mathbf{R}) \phi_{1,<}\left(\mathbf{R}^{\prime}\right) \phi_{1,<}^{*}\left(\mathbf{R}^{\prime}\right) \times \\
\left\langle\phi_{2,>}^{*}(\mathbf{R}) \phi_{2,>}\left(\mathbf{R}^{\prime}\right)\right\rangle_{0,>}\left\langle\phi_{2,>}^{*}\left(\mathbf{R}^{\prime}\right) \phi_{2,>}(\mathbf{R})\right\rangle_{0,>}, \\
2 V_{12}^{2} \iint d^{3} \mathbf{R} d^{3} \mathbf{R}^{\prime} \phi_{1,<}\left(\mathbf{R}^{\prime}\right) \phi_{1,<}^{*}(\mathbf{R}) \phi_{2,<}\left(\mathbf{R}^{\prime}\right) \phi_{2,<}^{*}(\mathbf{R}) \times \\
\left\langle\phi_{1,>}^{*}\left(\mathbf{R}^{\prime}\right) \phi_{1,>}(\mathbf{R})\right\rangle_{0,>}\left\langle\phi_{2,>}^{*}\left(\mathbf{R}^{\prime}\right) \phi_{2,>}(\mathbf{R})\right\rangle_{0,>}, \\
\left.2 V_{12}^{2} \iint_{d^{3} \mathbf{R} d^{3} \mathbf{R}^{\prime} \phi_{1,<}\left(\mathbf{R}^{\prime}\right) \phi_{1,<}^{*}(\mathbf{R}) \phi_{2,<}\left(\mathbf{R}^{*} \phi_{2,<}^{*}\left(\mathbf{R}^{\prime}\right) \times\right.}\left(\mathbf{R}^{\prime}\right) \phi_{1,>}(\mathbf{R})\right\rangle_{0,>}\left\langle\phi_{2,>}^{*}(\mathbf{R}) \phi_{2,>}\left(\mathbf{R}^{\prime}\right)\right\rangle_{0,>} \cdot \\
\left\langle\phi_{1,>}^{*}(\mathrm{~A} 23)\right.
\end{aligned}
$$

Analogous to the first-order calculation, the $V_{12^{-}}$ interaction mixes in corrections from $V_{0,1}$ to $V_{0,2}$ and vice versa (A20,A21). The group of terms proportional to $V_{0,1} V_{0,2}$ does not contain any connected diagrams and therefore does not contribute to the flow equations. This can be easily seen, since a nonzero mixing interaction $V_{12}$ is needed for the two Bose gases to have any influence on one another.

The last two groups of terms we have to consider are those proportional to $V_{0, i} V_{12}$. The connected diagrams are:

$$
\begin{aligned}
4 \frac{V_{0, i} V_{12}}{2} & \iint d^{3} \mathbf{R} d^{3} \mathbf{R}^{\prime} \phi_{i,<}(\mathbf{R}) \phi_{i,<}^{*}(\mathbf{R}) \phi_{\bar{i},<}\left(\mathbf{R}^{\prime}\right) \phi_{\bar{i},<}^{*}\left(\mathbf{R}^{\prime}\right) \times \\
& \left\langle\phi_{i,>}^{*}(\mathbf{R}) \phi_{i,>}\left(\mathbf{R}^{\prime}\right)\right\rangle_{0,>}\left\langle\phi_{i,>}^{*}\left(\mathbf{R}^{\prime}\right) \phi_{i,>}(\mathbf{R})\right\rangle_{0,>} .
\end{aligned}
$$

These corrections to $V_{12}$ appear twice in the cumulant expansion (A16).

One last complication arises in evaluating the corrections (A18-A24), since these corrections include non-local interactions not present in the original free energy functional. Assuming the interaction to be short-ranged, these non-local terms can be neglected within the $\varepsilon$ expansion [61]. Then, the second-order corrections take the form:

$$
\begin{aligned}
& \left\langle\phi_{i,>}^{*}(\mathbf{R}) \phi_{i,>}\left(\mathbf{R}^{\prime}\right)\right\rangle_{0,>}\left\langle\phi_{j,>}^{*}\left(\mathbf{R}^{\prime}\right) \phi_{j,>}(\mathbf{R})\right\rangle_{0,>} \\
\simeq & \int_{\Lambda / b}^{\Lambda} \frac{d \mathbf{k}}{(2 \pi)^{d}}\left(\frac{k_{B} T}{\varepsilon_{i, \mathbf{k}}-\mu_{i}}\right)\left(\frac{k_{B} T}{\varepsilon_{j, \mathbf{k}}-\mu_{j}}\right) .
\end{aligned}
$$

Now we are in the position to quantify the change in $V_{0,1}$, $V_{0,2}$ and $V_{12}$ after one RG step. As before, we rescale the Fourier components and integrate out infinitesimally thin 
(a)

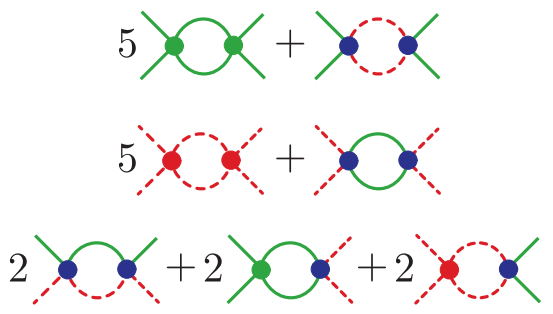

Figure 11: The second-order corrections of the $R G$ approach to (a) $V_{0,1}$, (b) $V_{0,2}$ and $V_{12}$.

shells in Fourier space:

$$
\begin{aligned}
d V_{0, i}= & -5 V_{0, i}^{2} \frac{\Lambda^{d}}{(2 \pi)^{d}} \frac{2 \pi^{\frac{d}{2}}}{\Gamma\left(\frac{d}{2}\right)} \frac{k_{B} T \cdot e^{-l d} d l}{\left(e^{-2 l} \varepsilon_{i, \Lambda}-\mu_{i}\right)^{2}} \\
& -V_{12}^{2} \frac{\Lambda^{d}}{(2 \pi)^{d}} \frac{2 \pi^{\frac{d}{2}}}{\Gamma\left(\frac{d}{2}\right)} \frac{k_{B} T \cdot e^{-l d} d l}{\left(e^{-2 l} \varepsilon_{\bar{i}, \Lambda}-\mu_{\bar{i}}\right)^{2}}, \quad(\mathrm{~A} 26) \\
d V_{12}= & -2 V_{12}^{2} \frac{\Lambda^{d}}{(2 \pi)^{d}} \frac{2 \pi^{\frac{d}{2}}}{\Gamma\left(\frac{d}{2}\right)} \frac{k_{B} T \cdot e^{-l d} d l}{\left(e^{-2 l} \varepsilon_{1, \Lambda}-\mu_{1}\right)\left(e^{-2 l} \varepsilon_{2, \Lambda}-\mu_{2}\right)} \\
& -2 V_{0,1} V_{12} \frac{\Lambda^{d}}{(2 \pi)^{d}} \frac{2 \pi^{\frac{d}{2}}}{\Gamma\left(\frac{d}{2}\right)} \frac{k_{B} T \cdot e^{-l d} d l}{\left(e^{-2 l} \varepsilon_{1, \Lambda}-\mu_{1}\right)^{2}} \\
& -2 V_{0,2} V_{12} \frac{\Lambda^{d}}{(2 \pi)^{d}} \frac{2 \pi^{\frac{d}{2}}}{\Gamma\left(\frac{d}{2}\right)} \frac{k_{B} T \cdot e^{-l d} d l}{\left(e^{-2 l} \varepsilon_{2, \Lambda}-\mu_{2}\right)^{2}} . \quad \text { (A27) }
\end{aligned}
$$

These second-order corrections are depicted in Fig. 11.

Finally, to obtain the flow equations, we have to rescale the chemical potentials and the coupling constants according to (A14):

$$
\begin{aligned}
\frac{d V_{0, i}}{d l}= & (4-d) V_{0, i}-5 V_{0, i}^{2} \frac{\Lambda^{d}}{(2 \pi)^{d}} \frac{2 \pi^{\frac{d}{2}}}{\Gamma\left(\frac{d}{2}\right)} \frac{k_{B} T}{\left(\varepsilon_{i, \Lambda}-\mu_{i}\right)^{2}} \\
& -V_{12}^{2} \frac{\Lambda^{d}}{(2 \pi)^{d}} \frac{2 \pi^{\frac{d}{2}}}{\Gamma\left(\frac{d}{2}\right)} \frac{k_{B} T}{\left(\varepsilon_{\bar{i}, \Lambda}-\mu_{\bar{i}}\right)^{2}}, \\
\frac{d V_{12}}{d l}= & (4-d) V_{12} \\
& -2 V_{12}^{2} \frac{\Lambda^{d}}{(2 \pi)^{d}} \frac{2 \pi^{\frac{d}{2}}}{\Gamma\left(\frac{d}{2}\right)} \frac{k_{B} T}{\left(\varepsilon_{1, \Lambda}-\mu_{1}\right)\left(\varepsilon_{2, \Lambda}-\mu_{2}\right)} \\
& -2 V_{0,1} V_{12} \frac{\Lambda^{d}}{(2 \pi)^{d}} \frac{2 \pi^{\frac{d}{2}}}{\Gamma\left(\frac{d}{2}\right)} \frac{k_{B} T}{\left(\varepsilon_{1, \Lambda}-\mu_{1}\right)^{2}} \\
& -2 V_{0,2} V_{12} \frac{\Lambda^{d}}{(2 \pi)^{d}} \frac{2 \pi^{\frac{d}{2}}}{\Gamma\left(\frac{d}{2}\right)} \frac{k_{B} T}{\left(\varepsilon_{2, \Lambda}-\mu_{2}\right)^{2}} .
\end{aligned}
$$

Setting $\mu_{1}=\mu_{2}=\mu$ and $V_{0,1}=V_{0,2}=V_{0}$ results in the RG equations for the intra- and inter-component interaction parameters. We rewrite the flow equations in terms of dimensionless variables, which are introduced in the main text:

$$
\begin{aligned}
\frac{d \mu_{\Lambda}}{d l} & =2 \mu_{\Lambda}-2 g_{0} \frac{T_{\Lambda}}{1-\mu_{\Lambda}}-g_{12} \frac{T_{\Lambda}}{1-\mu_{\Lambda}} \\
\frac{d g_{0}}{d l} & =\varepsilon g_{0}-5 g_{0}^{2} \frac{T_{\Lambda}}{\left(1-\mu_{\Lambda}\right)^{2}}-g_{12}^{2} \frac{T_{\Lambda}}{\left(1-\mu_{\Lambda}\right)^{2}}, \\
\frac{d g_{12}}{d l} & =\varepsilon g_{12}-2 g_{12}^{2} \frac{T_{\Lambda}}{\left(1-\mu_{\Lambda}\right)^{2}}-4 g_{0} g_{12} \frac{T_{\Lambda}}{\left(1-\mu_{\Lambda}\right)^{2}}
\end{aligned}
$$

We determine the three fixed points of these equations to be $\left(\mu_{\Lambda}^{*}, g_{0}^{*}, g_{12}^{*}\right)=(0,0,0),\left(\varepsilon /(5+\varepsilon), 5 \varepsilon /\left((5+\varepsilon)^{2} T_{\Lambda}\right), 0\right)$ and $\left(\varepsilon /(4+\varepsilon), 8 \varepsilon /\left(3(4+\varepsilon)^{2} T_{\Lambda}\right), 8 \varepsilon /\left(3(4+\varepsilon)^{2} T_{\Lambda}\right)\right.$. We Taylor-expand $1 /\left(1-\mu_{\Lambda}\right)$ such that the corrections in the flow equations are consistently quadratic in the coupling constants $[57,60]$. Since $\varepsilon_{\Lambda}$ serves as the maximum energy scale in the theory description, i.e. $\mu_{\Lambda} \ll 1$, the approach is justified, and the approximate RG equations take the form (18-20). Compared to the original flow equations (A30-A31), the second and the third fixed point is slightly shifted for these flow equations. However, we find that the nature of the fixed points, including the number of relevant, irrelevant and marginal scaling fields, remains unchanged [60].

\section{Appendix B: Expansion around fixed points to first order}

In this Appendix, we investigate the flow equations (18-20) in the vicinity of the fixed points. For that we Taylor-expand the flow equations to first order around them. For each fixed point the three resulting coupled linear differential equations can be solved analytically and the results are sketched qualitatively in Fig. 4. The coupled differential equations can be summarized by a $3 \times 3$ matrix. When we diagonalize this matrix, we can identify the so-called scaling fields $v_{\alpha}(\alpha=1,2,3)$, directions in the flow diagram that obey a particularly easy relation near the fixed points

$$
v_{\alpha}(l) \propto e^{\lambda_{\alpha} l},
$$

with the eigenvalues $\lambda_{\alpha}$. When $\lambda_{\alpha}>0$, the flow will be pushed away from the fixed point along the corresponding direction and the scaling field is said to be relevant. When $\lambda_{\alpha}<0$, the flow is attracted by the fixed point and the corresponding scaling field is said to be irrelevant. In the case when $\lambda_{\alpha}=0$, the scaling field is said to be marginal along the corresponding direction and the Taylor expansion up to first order is inconclusive.

For the first (trivial) fixed point (21) Taylor-expansion to first order results in the following differential equa- 
tions:

$$
\begin{aligned}
\frac{d \Delta \mu_{\Lambda}}{d l} & =2 \Delta \mu_{\Lambda}-2 T_{\Lambda} \Delta g_{0}-T_{\Lambda} \Delta g_{12}, \\
\frac{d \Delta g_{0}}{d l} & =\varepsilon \Delta g_{0}, \\
\frac{d \Delta g_{12}}{d l} & =\varepsilon \Delta g_{12} .
\end{aligned}
$$

Here, $\Delta \mu_{\Lambda}, \Delta g_{0}$ and $\Delta g_{12}$ are the deviations of the coupling constant from their fixed point values $\mu_{\Lambda}^{*}, g_{0}^{*}$ and $g_{12}^{*}$ :

$$
\begin{aligned}
\Delta \mu_{\Lambda} & =\mu_{\Lambda}-\mu_{\Lambda}^{*}, \\
\Delta g_{0} & =g_{0}-g_{0}^{*}, \\
\Delta g_{12} & =g_{12}-g_{12}^{*} .
\end{aligned}
$$

The eigenvalues and the corresponding scaling fields are:

$$
\begin{aligned}
& \lambda_{1}=2, \quad v_{1}=\Delta \mu_{\Lambda}, \\
& \lambda_{2}=\varepsilon, \quad v_{2}=\frac{2 T_{\Lambda}}{2-\varepsilon} \Delta \mu_{\Lambda}+\Delta g_{0}, \\
& \lambda_{3}=\varepsilon, \quad v_{3}=\frac{T_{\Lambda}}{2-\varepsilon} \Delta \mu_{\Lambda}+\Delta g_{12} .
\end{aligned}
$$

It follows that the first fixed point possesses three relevant scaling fields. Therefore, it is an unstable fixed point pushing the flow away from it.

First-order Taylor-expansion around the second fixed point (22) results in:

$$
\begin{aligned}
\frac{d \Delta \mu_{\Lambda}}{d l}= & 2\left(1-\frac{\varepsilon}{5}\right) \Delta \mu_{\Lambda}-2 T_{\Lambda}\left(1+\frac{\varepsilon}{5}\right) \Delta g_{0} \\
& -T_{\Lambda}\left(1+\frac{\varepsilon}{5}\right) \Delta g_{12}, \\
\frac{d \Delta g_{0}}{d l}= & -\varepsilon \Delta g_{0}, \\
\frac{d \Delta g_{12}}{d l}= & \frac{1}{5} \varepsilon \Delta g_{12} .
\end{aligned}
$$

$$
\begin{aligned}
\lambda_{1}=\frac{2(5-\varepsilon)}{5}, & v_{1}=\Delta \mu_{\Lambda}, \\
\lambda_{2}=-\varepsilon, & v_{2}=\frac{2(5+\varepsilon) T_{\Lambda}}{10+3 \varepsilon} \Delta \mu_{\Lambda}+\Delta g_{0}, \\
\lambda_{3}=\frac{\varepsilon}{5}, & v_{3}=\frac{(5+\varepsilon) T_{\Lambda}}{10-3 \varepsilon} \Delta \mu_{\Lambda}+\Delta g_{12} .
\end{aligned}
$$

The second fixed point has two relevant and one irrelevant scaling fields. Hence, it is a stable fixed point and the critical surface on which the flow is directed towards it is one-dimensional.

For the third fixed point (23) first-order expansion gives:

$$
\begin{aligned}
\frac{d \Delta \mu_{\Lambda}}{d l}=2\left(1-\frac{\varepsilon}{4}\right) \Delta \mu_{\Lambda}-2 T_{\Lambda}\left(1+\frac{\varepsilon}{4}\right) \Delta g_{0} \\
-T_{\Lambda}\left(1+\frac{\varepsilon}{4}\right) \Delta g_{12} \\
\frac{d \Delta g_{0}}{d l}=-\frac{2}{3} \varepsilon \Delta g_{0}-\frac{1}{3} \varepsilon \Delta g_{12} \\
\frac{d \Delta g_{12}}{d l}=-\frac{2}{3} \varepsilon \Delta g_{0}-\frac{1}{3} \varepsilon \Delta g_{12} . \\
\lambda_{1}=\frac{4-\varepsilon}{2}, \quad v_{1}=\Delta \mu_{\Lambda} \\
\lambda_{2}=-\varepsilon, \quad v_{2}=\frac{3 T_{\Lambda}}{2} \Delta \mu_{\Lambda}+\Delta g_{0}+\Delta g_{12} \\
\lambda_{3}=0, \quad v_{3}=-\frac{1}{2} \Delta g_{0}+\Delta g_{12} .
\end{aligned}
$$

The third fixed point possesses one relevant, one irrelevant and one marginal scaling field. Thus, the third fixed point is stable. The exact nature of the marginal direction of flow and the dimensionality of the critical surface are investigated in the main text.
[1] M. H. Anderson, J. R. Ensher, M. R. Matthews, C. E. Wieman, and E. A. Cornell, Science 269, 198 (1995).

[2] K. B. Davis, M. O. Mewes, M. R. Andrews, N. J. van Druten, D. S. Durfee, D. M. Kurn, and W. Ketterle, Phys. Rev. Lett. 75, 3969 (1995).

[3] C. C. Bradley, C. A. Sackett, J. J. Tollett, and R. G. Hulet, Phys. Rev. Lett. 75, 1687 (1995).

[4] M. Lewenstein, A. Sanpera, V. Ahufinger, B. Damski, A. Sen(De), and U. Sen, Advances in Physics 56, 243 (2007).

[5] I. Bloch, J. Dalibard, and W. Zwerger, Rev. Mod. Phys. 80, 885 (2008).

[6] A. Browaeys, H. Häffner, C. McKenzie, S. L. Rolston, K. Helmerson, and W. D. Phillips, Phys. Rev. A 72, 053605 (2005).

[7] A. Isacsson and S.M. Girvin, Phys. Rev. A 72, 053604 (2005).
[8] W. V. Liu and C. Wu, Phys. Rev. A 74, 013607 (2006).

[9] A. B. Kuklov, Phys. Rev. Lett. 97, 110405 (2006).

[10] T. Müller, S. Fölling, A. Widera, and I. Bloch, Phys. Rev. Lett. 99, 200405 (2007).

[11] L.-K. Lim, C. M. Smith, and A. Hemmerich, Phys. Rev. Lett. 100, 130402 (2008).

[12] V. M. Stojanović, C. Wu, W. V. Liu, and S. Das Sarma, Phys. Rev. Lett. 101, 125301 (2008).

[13] C. Wu, Modern Physics Letters B 23, 1 (2009).

[14] Q. Zhou, J. V. Porto, and S. Das Sarma, Phys. Rev. A 84, 031607 (2011).

[15] X. Li, E. Zhao, and W. V. Liu, Phys. Rev. A 83, 063626 (2011).

[16] Z. Cai and C. Wu, Phys. Rev. A 84, 033635 (2011).

[17] M. Ölschläger, G. Wirth, and A. Hemmerich, Phys. Rev. Lett. 106, 015302 (2011).

[18] P. Soltan-Panahi, D.-S. Luhmann, J. Struck, P. Win- 
dassinger, and K. Sengstock, Nat. Phys. 8, 71 (2012).

[19] X. Li, Z. Zhang, and W. V. Liu, Phys. Rev. Lett. 108, 175302 (2012).

[20] G. Wirth, M. M. Ölschläger, and A. Hemmerich, Nat. Phys. 7, 147 (2011)

[21] M. Lewenstein and W. V. Liu, Nat. Phys. 7, 101 (2011).

[22] M. Ölschläger, T. Kock, G. Wirth, A. Ewerbeck, C. Morais Smith and A. Hemmerich, New J. Phys. 15 083041 (2013).

[23] T. Kock, M. Ölschläger, A. Ewerbeck, W.-M. Huang, L. Mathey, and A. Hemmerich, Phys. Rev. Lett. 114, 115301 (2015).

[24] A. Eckardt, C. Weiss, and M. Holthaus, Phys. Rev. Lett. 95, 260404 (2005)

[25] N. Gemelke, E. Sarajlic, Y. Bidel, S. Hong and S. Chu, Phys. Rev. Lett. 95, 170404 (2005).

[26] H. Lignier, C. Sias, D. Ciampini, Y. Singh, A. Zenesini, O. Morsch, and E. Arimondo, Phys. Rev. Lett. 99, 220403 (2007).

[27] A. Zenesini, H. Lignier, D. Ciampini, O. Morsch, and E. Arimondo, Phys. Rev. Lett. 102, 100403 (2009).

[28] C. V. Parker, L.-C. Ha and C. Chin, Nature Physics 9, 769 (2013).

[29] L.-C. Ha, L. W. Clark, C. V. Parker, B. M. Anderson, and C. Chin, Phys. Rev. Lett. 114, 055301 (2015).

[30] A. Eckardt, P. Hauke, P. Soltan-Panahi, C. Becker, K. Sengstock, M. Lewenstein, Europhysics Lett. 89, 10010 (2010)

[31] J. Struck, C. Ölschläger, R. Le Targat, P. Soltan-Panahi, A. Eckardt, M. Lewenstein, P. Windpassinger, and K. Sengstock, Science 333, 996 (2011).

[32] K. Jimenez-Garcia, L. J. LeBlanc, R. A. Williams, M. C. Beeler, A. R. Perry, and I. B. Spielman, Phys. Rev. Lett. 108, 225303 (2012),

[33] J. Struck, C. Ölschläger, M. Weinberg, P. Hauke, J. Simonet, A. Eckardt, M. Lewenstein, K. Sengstock, and P. Windpassinger, Phys. Rev. Lett. 108, 225304 (2012).

[34] J. Struck, M. Weinberg, C. Ölschläger, P. Windpassinger, J. Simonet, K. Sengstock, R. Höppner, P. Hauke, A. Eckardt, M. Lewenstein, L. Mathey, Nat. Phys. 9, 738 (2013).

[35] Xiao-Gang Wen, Quantum Field Theory of Many-body Systems. (Oxford University Press, 2007).

[36] C. Becker, P. Soltan-Panahi, J. Kronjäger, S. Dörscher, K. Bongs and K. Sengstock, New J. Phys. 12, 065025 (2010).
[37] M. Y. Choi and S. Doniach, Phys. Rev. B 31, 4516 (1985).

[38] M. Yosefin and E. Domany, Phys. Rev. B 32, 1778 (1985).

[39] M. Hasenbusch, A. Pelissetto and E. Vicari, J. Stat. Mech. P12002 (2005)

[40] M. P. Zaletel, S. A. Parameswaran, A. Ruegg, and E. Altman, Phys. Rev. B 89, 155142 (2014).

[41] K. Huang, C. N. Yang, and J. M. Luttinger, Phys. Rev. 105, 776 (1957).

[42] H. T. C. Stoof, D. B. M. Dickerscheid, K. Gubbels, Ultracold Quantum Fields (Springer; 2009 edition)

[43] H.-P. Breuer, W. Huber, and F. Petruccione, Phys. Rev. E 61, 4883 (2000).

[44] A. Russomanno, A. Silva and G. E. Santoro, Phys. Rev. Lett 109, 257201 (2012)

[45] D. W. Hone, R. Ketzmerick, and W. Kohn, Phys. Rev. E 79, 051129 (2009).

[46] T. Iadecola, C. Chamon, R. Jackiw, and S.-Y. Pi, Phys. Rev. B 88, 104302 (2013)

[47] L D'Alessio and M. Rigol, Phys. Rev. X 4, 041048 (2014).

[48] A. Lazarides, A. Das, and R. Moessner, Phys. Rev. E 90, 012110 (2014).

[49] T. Shirai, T. Mori, and S. Miyashita, Phys. Rev. E 91, 030101(R) (2015)

[50] D. E. Liu, Phys. Rev. B 91, 144301 (2015)

[51] P. Ponte, A. Chandran, Z. Papić, D. A. Abanin, Annals of Physics 353, 196 (2015)

[52] D. A. Abanin, W. De Roeck, and F. Huveneers, Phys. Rev. Lett. 115, 256803 (2015).

[53] T. Mori, T. Kuwahara, and K. Saito, Phys. Rev. Lett. 116, 120401 (2016).

[54] T. Kuwahara, T. Mori, and K. Saito, Annals of Physics. 367, 96 (2016)

[55] M. Bukov, S. Gopalakrishnan, M. Knap, and E. Demler, Phys. Rev. Lett. 115, 205301 (2015).

[56] M. Bukov, M. Kolodrubetz, and A. Polkovnikov, Phys. Rev. Lett. 116, 125301 (2016)]

[57] E. Domany, D. Mukamel, and M. E. Fisher, Phys. Rev. B 15, 5432 (1977).

[58] P. R. Johnson et. al., New Journal of Physics 11, 093022 (2009).

[59] S. Will et. al., Nature 465, 197 (2010).

[60] J. Rudnick, Phys. Rev. B 18, 1406 (1978).

[61] K. G. Wilson and J. Kogut, Physics Reports 12, 75 (1974). 